\title{
Artificial Neural Networks Applied to Weekly Prediction of the Settlement Price of Differences in the Energy Market
}

\author{
Michele Goliath Martins de Araujo*, Leonardo Rocha Olivi* e Luís Henrique Lopes Lima* \\ *Engenharia Elétrica, Departamento de Energia, Universidade Federal de Juiz de Fora, \\ Caixa Postal, Juiz de Fora, MG, Brazil (e-mail: michele.goliath@engenharia.ufjf.br)
}

\begin{abstract}
The Settlement Price of Differences (SPD) is one of the main variables in the Electric Energy Free Market, and is disclosed by the Electric Energy Trading Chamber. Its behavior is nonlinear and contaminated by random variables. For any market participant, knowing their behavior is imperative to define their energy strategies. This work consists in the development of a model for prediction of SPD in the Brazilian Short-Term Market. The model is based on Artificial Neural Networks delayed in time with Levenberg-Marquardt method with Momentum. As input variables, SPD and its explanatory variables were used, Subsystem Induced Natural Energy, Energy Exchange and Stored Energy. As an output, the future SPD is obtained one week ahead. The results show an efficient methodology for the prediction, with the monitoring of its tendencies.
\end{abstract}

Resumo: O Preço de Liquidação das Diferenças (PLD) é uma das principais variáveis no Mercado Livre de Energia Elétrica, e é divulgada pela Câmara de Comercialização de Energia Elétrica. Seu comportamento é não-linear e contaminado por variáveis aleatórias. Para qualquer participante do mercado, conhecer seu comportamento é imperativo para definir suas estratégias energéticas. Este trabalho consiste no desenvolvimento de um modelo para predição do PLD no Mercado de Curto Prazo Brasileiro. O modelo é baseado em Redes Neurais Artificiais atrasadas no tempo com método de Levenberg-Marquardt com Momentum. Como variáveis de entrada foram utilizados o PLD e suas variáveis explanatórias, a Energia Natural Afluente por Subsistema, o Intercâmbio de Energia e a Energia Armazenada. Como saída, obtêm-se o PLD futuro uma semana afrente. Os resultados mostram uma eficiente metodologia para a predição, com o acompanhamento de suas tendências.

Keywords: Price Settlement of Differences; Energy Market; Short-Term Market; Artificial Neural Networks; Stochastic Forecasting.

Palavras-chaves: Preço de Liquidação das Diferenças; Mercado de Energia; Mercado de Curto Prazo; Redes Neurais Artificiais; Predição Estocástica.

\section{INTRODUÇÃO}

A energia elétrica é, atualmente, a principal fonte de energia do mundo, sendo de extrema importância na indústria mundial, e seu valor gera impacto a todos os participantes da sociedade. No Brasil, por ter sua geração de energia elétrica predominantemente hidráulica, os preços da energia sofrem influência de fatores naturais e de como estes recursos variam ao longo do ano. Assim, é de vital importância a previsão de preços a fim de se obter segurança no fornecimento e lucros na comercialização de energia elétrica (CCEEa, 2019).

A Câmara de Comercialização de Energia Elétrica (CCEE) contabiliza as diferenças entre o que foi contratado e o que foi produzido ou consumido. As diferenças negativas ou positivas são liquidadas no Mercado de Curto Prazo (Mercado Spot) e valoradas ao Preço de Liquidação das Diferenças (PLD), sendo que este é definido semanalmente para cada patamar de carga e para cada um dos quatro submercados no Brasil, conforme Figura 1, tendo como base o Custo Marginal de Operação (CMO) que é limitado por um preço mínimo e por um preço máximo (CCEEb, 2019).
O preço das Liquidações da Diferenças (PLD) é usado para valorar as diferenças de energia apuradas no Mercado de Curto Prazo (MCP). Utiliza-se o PLD para precificar cada montante de energia diferente do que foi estabelecido em contrato. Como o seu preço depende de recursos naturais, o PLD possui grande volatilidade ao longo do ano. Para conseguir prever de maneira eficaz, foi utilizado Redes Neurais Artificiais, um método não linear altamente robusto e preciso (Reston Filho, 2014).

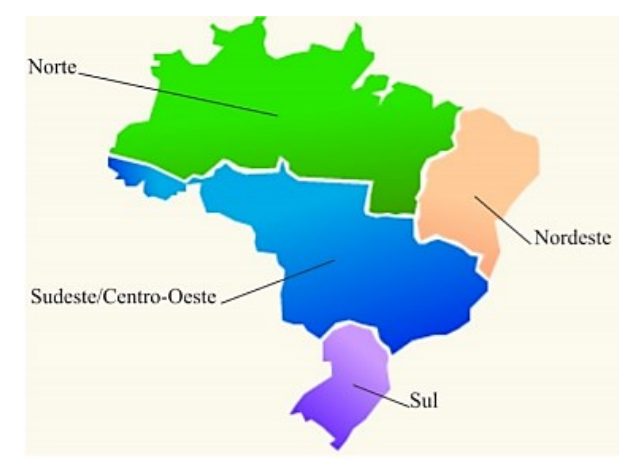

Figura 1 - Submercados do Sistema Elétrico Brasileiro, conforme mostrado pela CCEE. 
A predição do PLD é de importante conhecimento para o setor industrial e comercial, pois é uma decisão estratégica o momento de compra de energia, especialmente pela grande variabilidade a qual está sujeita. Um exemplo de alta volatilidade do PLD é observado na Figura 2 no período de janeiro de 2006 a janeiro de 2011, com a alta do preço do PLD, e grande variação entre os períodos (CCEEc, 2019).

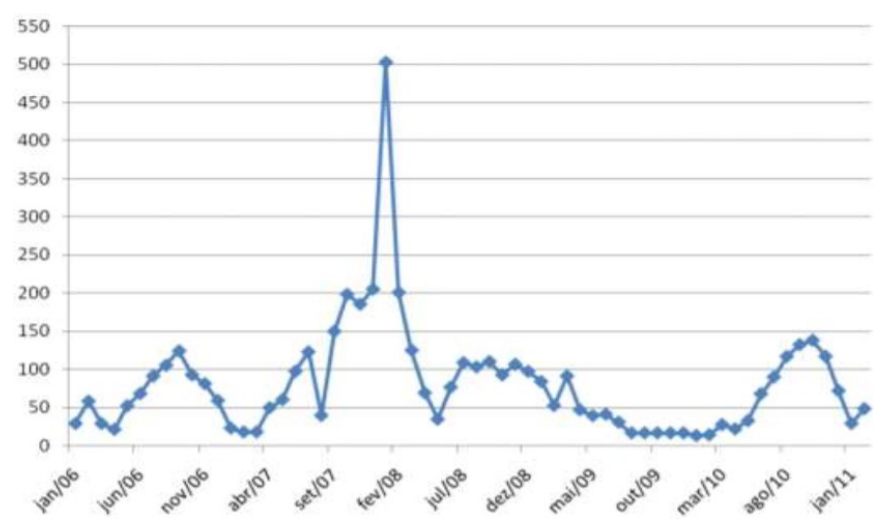

Figura 2 - Alta volatilidade do PLD (CCEEc, 2019).

Assim, o PLD é um importante instrumento de orientação dos agentes do mercado de energia elétrica, pois pode afetá-los financeiramente. Distribuidores e Comercializadores ficam expostos ao PLD por não terem contratado energia suficiente para seu suprimento; por outro lado, geradores que tenham contratado maior suprimento de energia do que conseguem fornecer. Nestas situações ficam expostos ao Mercado de Curto Prazo (MCP) (Azevedo, 2004; CCEEc, 2019).

Este trabalho se propõe a implementar um modelo baseado em Redes Neurais Artificiais para o aprendizado e predição do Preço de Liquidação das Diferenças no Mercado de Curto Prazo de Energia brasileiro. O presente trabalho se divide da seguinte maneira: a Seção 2 faz uma revisão bibliográfica do tema, a Seção 3 apresenta a metodologia escolhida para a predição, a Seção 4 apresenta os resultados e a Seção 5 as conclusões e trabalhos futuros.

\section{REVISÃO BIBLIOGRÁFICA}

O mercado spot é um segmento da Câmara de Comercialização de Energia Elétrica (CCEE) no qual são contabilizadas as diferenças entre os montantes de energia elétrica contratados pelos agentes e os montantes de geração e de consumo efetivamente verificados e atribuídos aos respectivos agentes (CCEEa., 2019).

Todos os contratos de compra e venda de energia celebrados no mercado, tanto no Ambiente de Contratação Regulado como no Ambiente de Contratação Livre devem ser registrados na CCEE, que realiza a medição dos montantes efetivamente produzidos/consumidos por cada agente. As diferenças apuradas, positivas ou negativas, são contabilizadas para posterior liquidação financeira no Mercado de Curto Prazo e valoradas ao Preço de Liquidação das Diferenças (PLD) (CCEEc.,2019; ONSa, 2019).
No novo modelo, a contratação de energia pode ser feita no Ambiente de Contratação Regulada (ACR) ou no Ambiente de Contratação Livre (ACL). Esses dois ambientes possuem diferenciações, quais sejam no primeiro (ACR) os consumidores ditos cativos partilham contratos de adesão com o distribuidor que opera na região, exemplo disso são as residências, lojas e pequenas indústrias, não podendo negociar as cláusulas do contrato.

Já no segundo modelo de contratação (ACL), os consumidores são ditos livres, pois podem escolher seu fornecedor de energia (não tendo limitações físicas da conexão com o sistema de energia elétrica), bem como qualquer dos vendedores presentes no Sistema Interligado Nacional (SIN), ademais nesse ambiente os preços e condições contratuais são negociáveis (Abraceel, 2017).

Dentre os estudos relacionados à modelagem e predição, diversas abordagens utilizando métodos e modelos matemáticos como forma de prever movimentações no mercado financeiro. Nas últimas décadas, essa foi uma área de grande interesse para a comunidade de Inteligência Artificial (Ceretta et al., 2010).

No trabalho de Pessanha (2007), o autor afirma que para se obter um melhor resultado na contratação de energia deve ser combinado métodos de otimização e simulação. Foi utilizado o método Sample Path Optimization ou Sample Avarege Aproximation para simular e obter a função objetivo e otimizou os resultados com o algoritmo genético Riskoptimizer da Palisade Corporation.

Em Mendonça (2008), o autor aborda o problema de contratação de energia elétrica em curto e longo prazo utilizando Redes Neurais Artificiais. Seu estudo está baseado na projeção de 5 anos propondo que a otimização na contratação de energia seja feita como uma estratégia da distribuidora, dentro dos seus limites regulatórios, já que o mercado apresenta desvios.

No trabalho de Campos (2008) foi utilizado a previsão de demanda abordando técnicas de inteligência computacional: ARIMA, NARIMA, Rede Neuro-Fuzzy (RNF) e RNA. Além disso, comparou os resultados por meio dos índices de desempenho mais usuais na área, o Mean Percentual Error (MPE), o Mean Absolute Percentual Error (MAPE) e Root Mean Squared Error (RMSE).

Reston Filho (2014) desenvolveu em seu trabalho uma coletânea de técnicas para o problema de predição de dados PLD no mercado brasileiro de energia elétrica, dentre as quais constam modelos de médias móveis e Redes Neurais Artificiais. $\mathrm{O}$ autor desenvolveu uma técnica híbrida, mesclando modelos ARIMA de médias móveis como entradas para redes neurais artificiais.

Desde então, os trabalhos desenvolvem metodologias parecidas, e resultados análogos. O objetivo deste trabalho é a previsão do preço do PLD com o tempo de uma semana à frente (no futuro), e, para isso, foram utilizados os modelos computacionais das Redes Neurais Artificiais (RNAs). Dentro das RNAs o método utilizado foi de LevenbergMarquardt associado ao termo de aceleração denominado Momentum em uma rede Perceptron Multicamadas. 


\section{METODOLOGIA}

As Redes Neurais Artificiais são modelos computacionais baseados no comportamento de neurônios biológicos, e podem aprender a relação entre entradas e saídas, em treinamentos supervisionados, com base nos dados de treinamento, sendo, portanto, amplamente utilizadas para modelagem, predição e classificação de dados, uma vez que possibilita a identificação de relações presentes em fontes de dados limitados, incompletos, com ruídos, dinâmicos e não lineares (Haykin, 2002).

Esses modelos computacionais admitem diversos tipos de configurações, sendo as mais utilizadas para os problemas de aprendizado de funções e predição de dados de séries temporais são as Perceptron Multicamadas, conforme mostrado na Figura 3. Nesse tipo de modelo computacional, o conhecimento acerca dos dados fica armazenado nos pesos $\omega$ que ligam cada um dos neurônios, denominados pesos sinápticos. A chave para um bom funcionamento de uma rede neural é a calibração desses pesos de maneira apropriada, pelos diversos tipos de treinamentos existentes na literatura (Haykin, 2002).

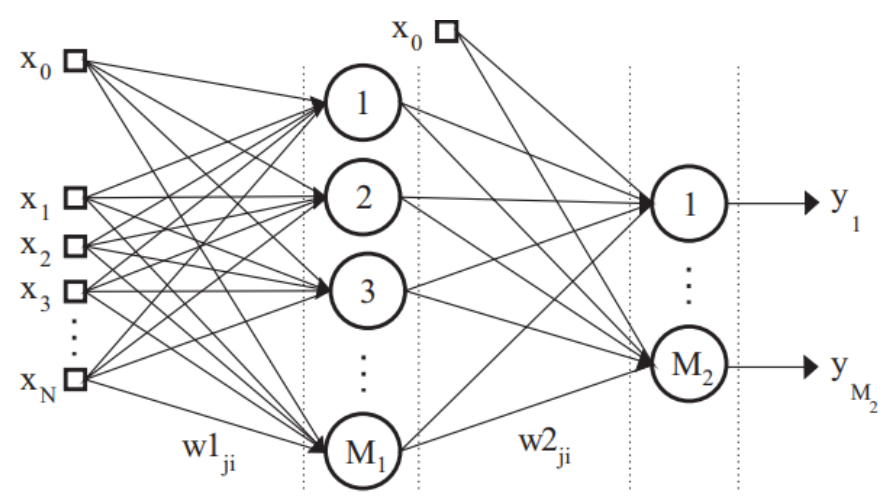

Figura 3 - Rede Perceptron Multicamadas utilizada.

Vale ressaltar que neste trabalho utilizou-se apenas uma única camada escondida, conforme mostrado na Figura 3, pois de acordo com o Teorema da Aproximação Universal de Kolmogorov e Cybenko, com apenas uma única camada escondida pode-se obter uma boa generalização e aproximação da rede (Haykin, 2002; Khanlari et al., 2012). Dados, portanto, um conjunto de pesos, é necessário determinar como alterá-los, a fím de reduzir o erro entre as saídas da rede e a série temporal em análise.

Levenberg-Marquardt é um método de segunda ordem classificado como quasi-Newton, uma vez que utiliza uma aproximação para as segundas derivadas dos erros, acelerando, assim, a convergência do treinamento. Esse método aproxima a segunda derivada multidimensional que é chamada de matriz Hessiana, por meio do Gradiente, conforme a Equação 1:

$$
H(E Q M) \cong \nabla E Q M^{T} \nabla E Q M
$$

em que EQM é o erro quadrático médio (Mean Squared Error, MSE), dado por:

$M S E=\sum_{i} \frac{1}{2}\left(d_{i}-y_{i}\right)^{2}$

em que $d$ são as saídas desejadas para a RNA, a própria série temporal, e $y$ é a saída da RNA, em que se deseja que o erro seja o menor possível. A aproximação é escolhida pois a mesma produz um bom direcionamento para o treinamento dos pesos sinápticos, sem a desvantagem do cálculo literal da Hessiana, que possui complexidade computacional. Assim, reduz-se o tempo de cômputo e consequente execução da estratégia.

O cálculo do Gradiente é dado com relação a cada um dos pesos sinápticos, e é uma relação bem conhecida na literatura. O termo de ajuste de cada um dos pesos é dado pelo termo de Levenberg-Marquardt:

$$
L M_{\omega}=\left(H_{\omega}+\sigma I\right)^{-1} \nabla E Q M_{\omega}^{T} E Q M
$$

em que $\omega$ identifica os pesos sinápticos da camada em questão, $\sigma$ é o termo de Levenberg-Marquardt, um valor pequeno para evitar problemas de singularidade e I é a matriz identidade de ordem apropriada. Assim, o ajuste dos pesos sinápticos de cada uma das camadas é dado na Equação 4 :

$w_{i}{ }^{\{t+1\}}=w_{i}{ }^{\{t\}}-\eta_{w i} L M_{\omega i}$

A configuração dos dados de entrada da RNA, adquiridos por meio da CCEE e do Operador Nacional do Sistema (ONS), é formada pelo PLD bem como por dados de armazenamento como a Energia Armazenada (EA), Energia Natural Afluente por subsistema (ENASub) e o Intercâmbio de Energia (IE), uma vez que estas são as variáveis citadas na literatura que possuem uma grande influência no PLD (Reston Filho, 2014; ONSb, 2019).

Os valores relacionados aos dados do PLD foram retirados do site do CCEE utilizando seu valor médio de carga, sendo todos esses dados do período de 31/03/2015 à 31/05/2017, totalizando 97 semanas, aproximadamente 1 ano e 11 meses, e considerando a semana operativa e em MWmédio da Região Sudeste/Centro-Oeste, uma vez que este trabalho se desenvolveu nesta região. A razão de se escolher essa quantidade de dados se dá pela constante atualização do sistema, tornando dados antigos desatualizados, bem como os valores mínimo e máximo do PLD são calculados anualmente. Assim, evita-se que dados muito antigos e obsoletos, provenientes de realidades socioeconômicas muito diferentes das atuais, contaminem as predições atuais.

A inserção dos dados de PLD e do ONS como entradas da rede neural precisam de uma estratégia de atraso no tempo, de modo que a rede receba as propriedades passadas dos dados, adquirindo propriedades autorregressivas, como as características de um filtro de resposta ao impulso finita (FIR). Dessa maneira, organizam-se os dados de maneira a fazer uma estratégia de atraso temporal denominada Time Delay Neural Network (TDNN) (Silva, 2010). Desta maneira, duas abordagens de entradas para as redes neurais serão implementadas, ambas dadas nas Equações 5 e 6. 
Conforme é possível observar, a recorrência para as variáveis do ONS foi utilizada na abordagem da Equação 5, enquanto que na Equação 6 tem-se a recorrência somente do PLD. A estratégia das Equações 5 e 6 se devem para verificação da necessidade da recorrência temporal das variáveis do ONS, uma vez que isto implicaria numa redução significativa do número de entradas da Equação 6 com relação à Equação 5 .

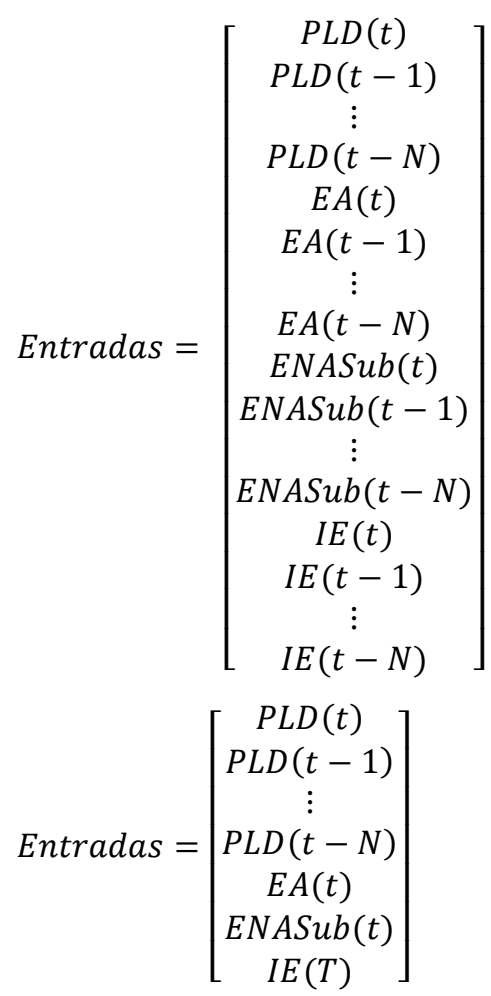

As saídas da rede neural, conforme descrito anteriormente, devem predizer o futuro imediato do PLD. Portanto, são configuradas conforme a Equação 7 para ambas as estratégias comentadas anteriormente.

Saída $=P L D(t+1)$

Desta forma, se define as configurações de entradas e saídas para a rede neural, de maneira a estabelecer um comparativo de estratégias a fim de verificar a influência das recorrências dos dados da ONS na predição do PLD.

\section{RESULTADOS}

Nesta seção serão apresentados os resultados de aprendizado e de predição para ambas as abordagens mencionadas, e suas respectivas análises. Como optou-se por utilizar neurônios com função de ativação sigmoide na camada de saída da RNA, uma normalização dos dados é necessária, e é dada por:

$n_{i}=n_{\text {max }}-\frac{\left(n_{\text {max }}-n_{\text {min }}\right)\left(s_{\text {max }}-s_{i}\right)}{\left(s_{\text {max }}-s_{\text {min }}\right)}$ em que $s$ representa os valores originais da série temporal e $n$ representa os valores normalizados. A sigmoide utilizada foi a função logística, portanto, $n_{\max }$ é 0,9 e $n_{\min }$ é 0,1 , sendo esses os valores de normalização escolhidos empiricamente.

\subsection{Abordagem de atraso para todas as variáveis de entrada}

A primeira abordagem é relativa à aplicação da estratégia TDNN, atraso temporal, para todas as variáveis de entrada escolhidas para a rede neural, conforme descrito na Equação 5, a saber, PLD bem como por dados de armazenamento como a Energia Armazenada (EA), Energia Natural Afluente por subsistema (ENASub) e o Intercâmbio de Energia (IE).

Diversas tentativas de calibração dos parâmetros foram realizadas, e a configuração mais benéfica para os dados obteve os seguintes parâmetros: 10 atrasos temporais para os dados; taxa de momentum de 0,9; com 95 neurônios na camada intermediária; o termo de Levenberg-Marquardt como 0,001 ; e a função de ativação com inclinação de 0,8 e deslocamento da média de 0,62 , valores calculados a partir do espalhamento dos dados.

O treinamento por meio de Levenberg-Marquardt com Momentum foi realizado por 300 iterações (épocas), dentro das quais a variação de aprendizado tornou-se suficientemente pequena para que a interrupção do treinamento fosse aceita. Conforme é possível observar na Figura 4, o nível de erro quadrático médio atingido foi de $M S E=0,062694$. Ainda na Figura 4 é possível observar a ação natural do método de treinamento de segunda ordem, Levenberg-Marquardt, que ocasiona uma grande variação no início do treinamento, mas depois as oscilações cessam, realizando-se, assim, a convergência do erro.

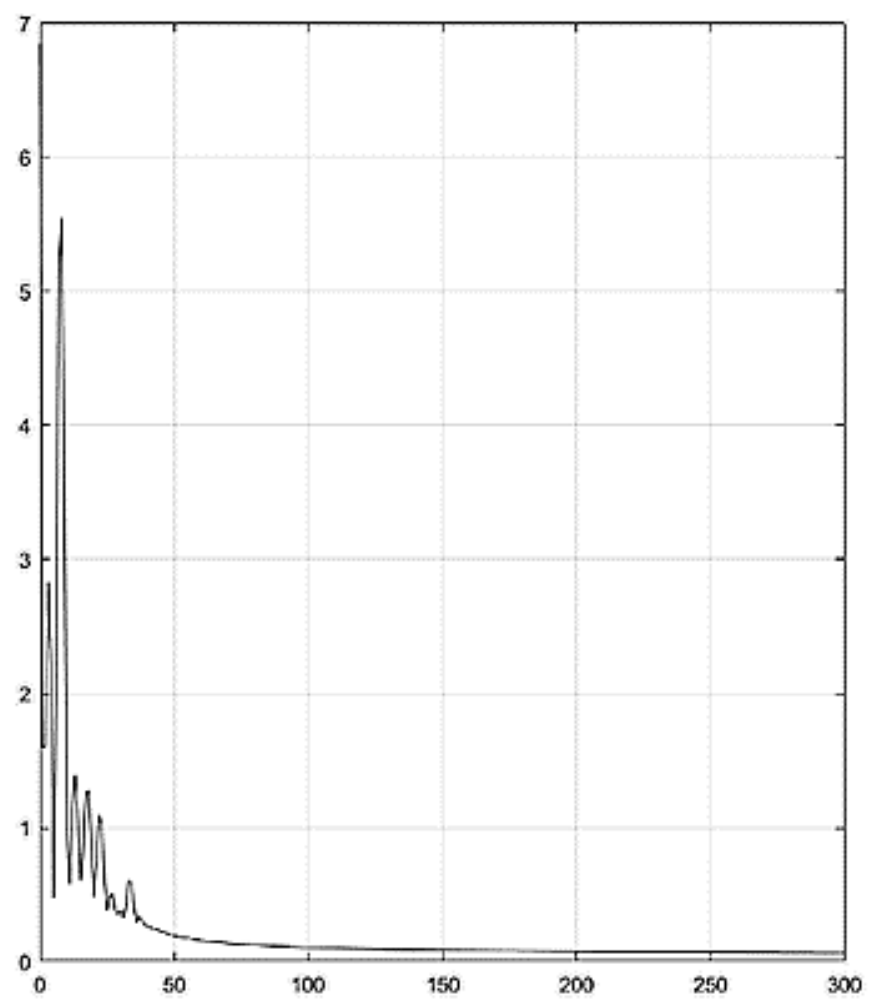

Figura 4 - Evolução do erro quadrático médio da seção 4.1. 
Ao final do treinamento, é possível observar o resultado da predição do algoritmo contendo a regressão dos dados de entrada, conforme disposto na Figura 5. É possível observar que a predição de um passo afrente, ou seja, de uma semana adiante, adquiriu a propriedade de generalizar a tendência dos dados, permitindo ao usuário da estratégia ter uma percepção acurada sobre a variação dos dados e, assim, planejar uma decisão acerca da compra de energia no mercado de curto prazo.

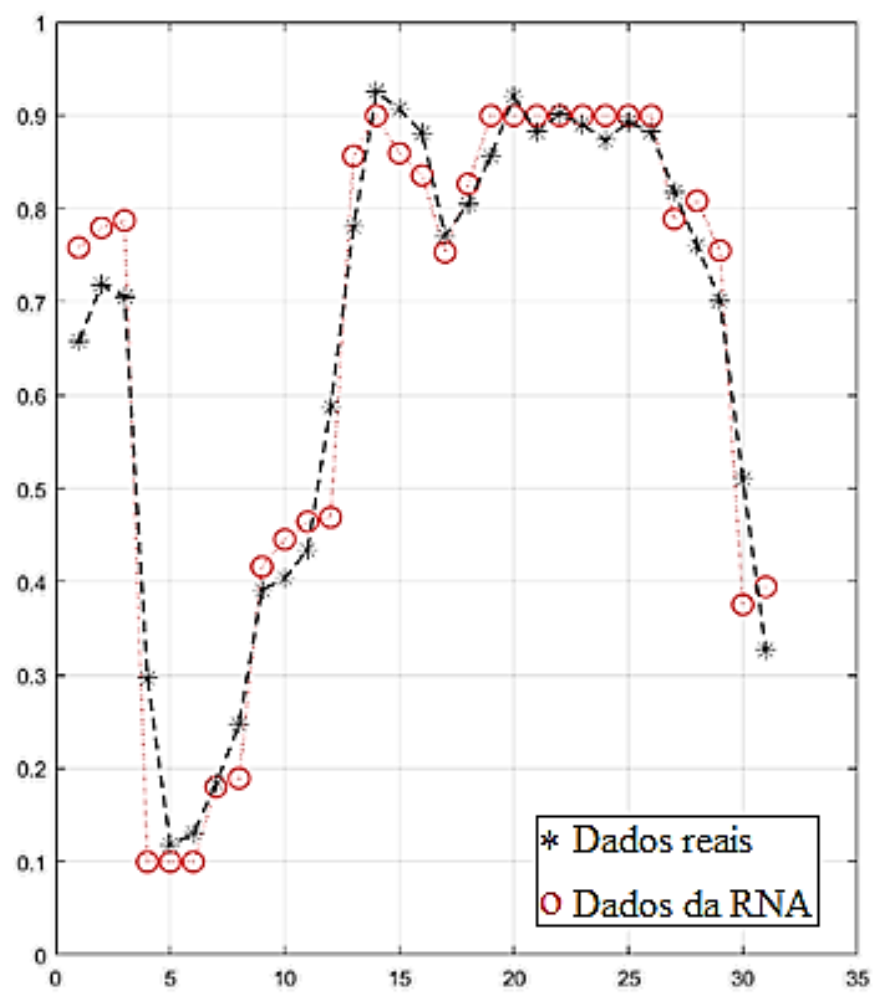

Figura 5 - Predição dos dados de PLD da seção 4.1.

\subsection{Abordagem de atraso somente para o PLD}

A segunda abordagem é relativa à aplicação de estratégia TDNN, atraso temporal, somente para a variável do PLD, conforme descrito na Equação 6. Assim, nesta abordagem as outras variáveis de entrada possuem seus dados utilizados no tempo presente, ou seja, o mesmo tempo do PLD com o dado no tempo atual.

Após a calibração dos parâmetros da rede neural e dos atrasos nos dados de entrada, a configuração mais vantajosa obteve os seguintes parâmetros: 30 atrasos temporais para os dados, número maior quando comparado à seção 4.1 , uma vez que menos dados de entrada estavam disponíveis para o aprendizado da rede; taxa de momentum de 0,95, para permitir uma aceleração de treinamento aproximadamente equiparada com a anterior; com 95 neurônios na camada intermediária; o termo de Levenberg-Marquardt como 0,001; e a função de ativação com inclinação de 0,8 e deslocamento da média de 0,62 , os mesmos valores calculados a partir do espalhamento dos dados.
Assim como no primeiro caso, o treinamento por meio de Levenberg-Marquardt com Momentum foi realizado por 300 iterações (épocas), em que também se observou a estabilização do erro, com uma variação de gradientes suficientemente pequena, permitindo assim a interrupção do treinamento, conforme é possível observar na Figura 6. Neste caso, o nível de erro quadrático médio atingido foi de $M S E=$ 0,005719. Ainda na Figura 6, é possível observar que, para este caso, a ação de treinamento não ofereceu tantas variações dinâmicas quanto no caso anterior, realizando-se, também, a convergência do erro.

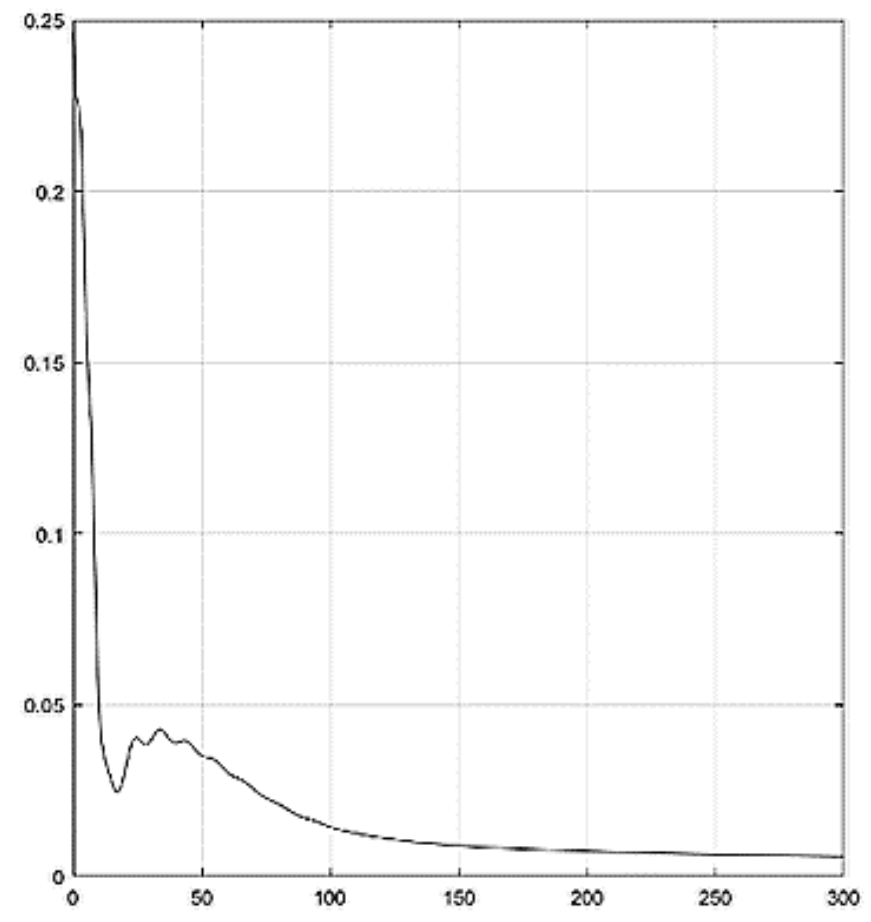

Figura 6 - Evolução do erro quadrático médio da seção 4.2.

É possível observar na Figura 7 que, no caso da predição dos dados, igualmente para uma semana no futuro, a rede também foi capaz de generalizar os dados, embora o maior número de atrasos temporais utilizados (no caso, 30 atrasos contra apenas 10 da seção anterior), disponibiliza para a predição uma quantidade menor de dados que na seção anterior. No entanto, os resultados são satisfatórios, fornecendo também uma boa estimativa dos preços de PLD futuros.

\section{CONCLUSÕES}

Este trabalho mostrou uma estratégia para a predição de um tipo de série temporal estocástica influente no mercado de energia de curto prazo brasileiro. Conforme é possível observar nas Figuras 5 e 7, ambas as abordagens tiveram um comportamento qualitativamente similar, apesar da diferença de dados generalizados e preditos entre ambas, o que mostra as variáveis explanatórias contidas no PLD. A quantidade de atrasos compensou a diferença de números de atrasos temporais nos dados explanatórios, reflexo da TDNN. 


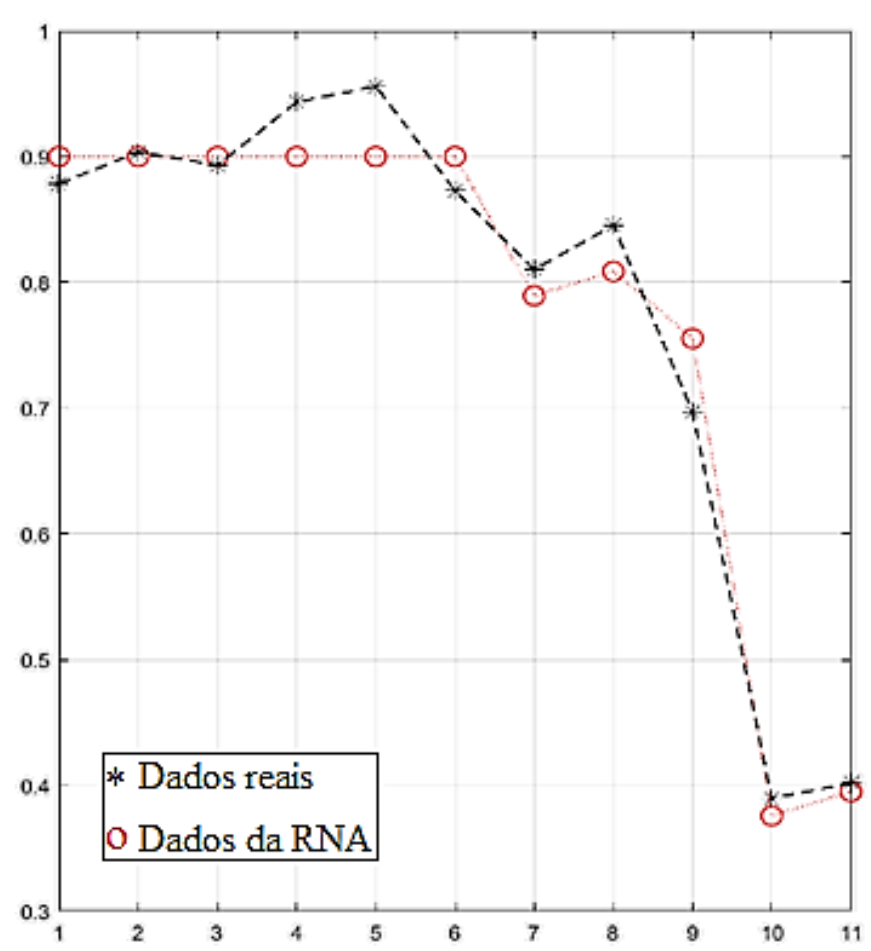

Figura 7 - Predição dos dados de PLD da seção 4.2.

Em termos quantitativos, a segunda abordagem mostrou um erro quadrático médio dez vezes menor. Apesar deste fato numérico, não significa que a segunda abordagem é necessariamente melhor, uma vez que a mesma possuiu uma quantidade substancialmente menor no momento de predição, tendo uma quantidade de dados substancialmente maior no treinamento. Se os dados de predição são numericamente menores, o cálculo do MSE é influenciado, o que pode mascarar os dados numéricos com um espalhamento menor da medida em questão.

Levando e consideração o desempenho do primeiro método sobre uma quantidade maior de dados, com um desempenho satisfatório, pode-se assumir que a primeira abordagem é preferível à segunda, pois atingiu um resultado qualitativo tão interessante quanto, porém sobre uma quantidade de dados três vezes maior, capturando a tendência dos dados com uma menor quantidade de atrasos temporais necessários para as variáveis de entrada, demonstrando que os dados existentes do ONS podem agregar informações acerca do PLD.

Os resultados demonstram que a metodologia foi capaz de capturar as tendências dos dados, sendo um bom indicativo da variação do PLD, auxiliando, consequentemente, aos usuários do mercado de curto prazo, a planejarem suas ações, maximizando seu sucesso nas tomadas de decisões. Os próximos passos envolvem a aplicação de técnicas de predição de longo prazo para as variáveis explanatórias, de modo a se obter maiores quantidades de tempos futuros preditos na estratégia. Uma vez que os dados explanatórios possuem fundamentalmente o mesmo problema estocástico, redes neurais são boas opções para a tarefa de predizê-los, conforme os dados deste trabalho evidenciam.

\section{REFERÊNCIAS}

ABRACEEL, Associação Brasileira dos Comercializadores de Energia (2017). Cartilha do Mercado Livre de Energia. Disponível em: $<$ http://www.abraceel.com.br/archives/files/Abraceel_Ca rtilha_MercadoLivre_V9.pdf $>$. Acesso em: 29/04/2019.

Azevedo, E.M. (2004). Modelo computacional de teoria dos jogos aplicado aos leilões brasileiros de energia elétrica. Tese de Doutorado. Universidade Estadual de Campinas.

Campos, R.J. (2008). Previsão de séries temporais com aplicações a séries de consumo de energia elétrica. Dissertação de Mestrado em Engenharia Elétrica. Universidade Federal de Minas Gerais, Minas Gerais.

CCEEa, Câmara de Comercialização de Energia Elétrica (2019). Disponível em: <http://www.ccee.org.br/>. Acesso em: 29/04/2019.

CCEEb, Câmara de Comercialização de Energia Elétrica (2019). Comercialização. Disponível em: $<$ http://www.ccee.org.br/portal/faces/pages_publico/ond e-atuamos/comercializacao? adf.ctrlstate $=$ gd9wkknpa_55\&_afrLoop $=412424689971715 \#$ ! $>$. Acesso em: 29/04/2019.

CCEEc, Câmara de Comercialização de Energia Elétrica (2019). Preços. Disponível em: $<$ http://www.ccee.org.br/portal/faces/oquefazemos_men u_lateral/leiloes?_afrLoop $=412702487533573 \& \_$adf.ctrl state $=$ gd9 wkknpa_116\#!\%40\%40\%3F_afrLoop\%3D412 702487533573\%26_adf.ctrl-state\%3Dgd9wkknpa_120>. Acesso em: 29/04/2019.

Haykin, S. (2001). Redes neurais: principios e prática. 2. ed. Porto Alegre: Bookman.

Khanlari, G.R.; Heidari, M.; Momeni, A.A.; Abdi, Y. (2012). Prediction of shear strength parameters of soils using artificial neural networks and multivariate regression methods. Engineering Geology, Amsterdam, v. 131/132, p. 11-18.

Mendonça, F.J.M. (2008). Análise do risco dos contratos de energia elétrica das concessionárias de distribuição. Dissertação de Mestrado em Engenharia Elétrica. Universidade Federal do Maranhão.

ONSa, Operador Nacional do Sistema Elétrico (2019). Disponível em: <http://ons.org.br/>. Acesso em: 29/04/2019.

ONSb, Operador Nacional do Sistema Elétrico (2019). Histórico da operação. Disponível em: $<$ http://ons.org.br/paginas/resultados-da-operacao/historicoda-operacao $>$. Acesso em: 29/04/2019.

Pessanha, A.J. (2007). Estratégia de contratação de energia elétrica em leilões regulados: aplicação de um modelo de simulação e otimização. Dissertação de Mestrado. Universidade Federal Fluminense.

Reston Filho, J.C. (2014). Previsão Multi-passos à frente do preço de energia elétrica de curto prazo no mercado brasileiro. Tese de Doutorado. Universidade Federal do Pará.

Silva, I.N. (2010). Redes Neurais Artificiais Para Engenharia E Ciências Aplicadas. Curso Prático, ArtLiber. 